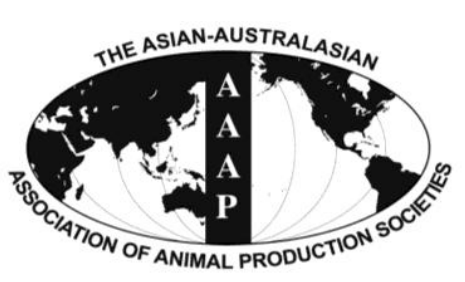

Open Access

Asian Australas. J. Anim. Sci.

Vol. 27, No. 9 : 1355-1359 September 2014

http://dx.doi.org/10.5713/ajas.2014.14046

www.ajas.info

pISSN 1011-2367 elSSN 1976-5517

\title{
Recombinant Goat VEGF164 Increases Hair Growth by Painting Process on the Skin of Shaved Mouse
}

\author{
Wenlei Bao ${ }^{\mathrm{a}}$, Jianxin Yin ${ }^{\mathrm{a}}$, Yan Liang, Zhixin Guo, Yanfeng Wang, \\ Dongjun Liu, Xiao Wang*, and Zhigang Wang* \\ College of Life Science, Inner Mongolia University, Hohhot, 010021, China
}

\begin{abstract}
To detect goat vascular endothelial growth factor (VEGF)-mediated regrowth of hair, full-length VEGF164 cDNA was cloned from Inner Mongolia cashmere goat (Capra hircus) into the pET-his prokaryotic expression vector, and the recombinant plasmid was transferred into $E$. coli BL21 cells. The expression of recombinant $6 \times$ his-gVEGF164 protein was induced by $0.5 \mathrm{mM}$ isopropyl thio- $\beta$-D-galactoside at $32^{\circ} \mathrm{C}$. Recombinant goat VEGF164 (rgVEGF164) was purified and identified by western blot using monoclonal anti-his and anti-VEGF antibodies. The rgVEGF164 was smeared onto the dorsal area of a shaved mouse, and we noted that hair regrowth in this area was faster than in the control group. Thus, rgVEGF164 increases hair growth in mice. (Key Words: Goat, Vascular Endothelial Growth Factor (VEGF), Recombinant Protein, Hair Growth)
\end{abstract}

\section{INTRODUCTION}

Vascular endothelial growth factor (VEGF) is a specific heparin-binding glycoprotein (Ferrara and Henzel, 1989) that is also known as vascular permeability factor (VPF) (Senger et al., 1993). The VEGF is a homodimer that comprises 2 polypeptide chains with a molecular mass of approximately $45 \mathrm{kDa}$ (Ferrara and Henzel, 1989; Gospodarowicz et al., 1989). The human VEGF gene has 8 exons that are separated by 7 introns. By alternative splicing, 7 VEGF transcripts are expressed in human, encoding polypeptides of 189, 165, 121, 145, 183,148, and 206 amino acids, respectively (Jingjing et al., 1999; Poltorak et al., 1997; Tischer et al., 1991; Whittle et al., 1999). The domain that is encoded by exons 1-5 and 8 are present in all VEGF splice variants. The VEGF206 contains all 8 peptideencoding exons. The VEGF189 and VEGF183 lack some of the peptides that are encoded by exon 6 . The VEGF165 lacks the peptides encoded by exons 6, VEGF148 lacks the

* Corresponding Authors: Zhigang Wang. Tel: +86-471-4995867 (8008), Fax: +86-471-4992435, E-mail: 1swzg@imu.edu.cn / Xiao Wang. E-mail: wxiao2008@gmail.com

${ }^{a}$ These authors contributed equally to this work.

Submitted Jan. 21, 2014; Revised Mar. 11, 2014; Accepted Mar. 31, 2014 peptides encoded by exon 6 and part of exon 7, while VEGF145 lacks the peptides encoded by exon 7 and part of exon 6, VEGF121 lacks the peptides encoded by both exons 6 and 7. The VEGF165 is secreted and binds to heparin, rendering it the most frequently studied splice variant.

The VEGF increases vascular permeability; promotes angiogenesis; and enhances survival, proliferation, and migration in various cell types. For example, the differentiation of endothelial cells and cancer cells is regulated by VEGF through an intracrine mechanism (Carmeliet et al., 1996; Ford and D'Amore, 2012; Gordon et al., 2012; Liu et al., 2012; Sitohy et al., 2012). The VEGF mediates vascular inflammation by regulating osteopontin expression ( $\mathrm{Li}$ et al., 2012c) and contributes to hair growth (Li et al., 2012b). Exogenous VEGF dose-dependently stimulates cell proliferation, which is mediated by vascular endothelial growth factor receptor 2 (VEGFR-2) through phosphorylation of extracellular signal-regulated kinase (ERK) in human outer root sheath cells and human hair follicle dermal papilla cells ( $\mathrm{Li}$ et al., 2012a; Magnuson et al., 2012). And VEGF expression in secondary hair follicles than it did in primary hair follicles (Zhang et al., 2013). The VEGF accelerates hair growth in mice and humans, but its function has not been determined in goat. 
To detect goat VEGF-mediated regrowth of hair, we cloned Inner Mongolia Cashmere goat VEGF164 gene (JX524883.1), which encodes a 190-amino-acid peptide with a signal peptide of 26 amino acids and shows a high homology to $V E G F$ genes in other vertebrates. We then expressed goat VEGF164 (gVEGF164) in E. coli and purified the rgVEGF164 recombinant protein to perform functional studies of gVEGF164. The rgVEGF164 was smeared across a dorsal area of a shaved mouse, and hair regrowth was monitored.

\section{MATERIAL AND METHODS}

\section{Molecular cloning of goat VEGF164 gene and transferred into $E$. coli}

Total RNA was isolated using RNAzol (RNAiso Plus, TaKaRa Co. Ltd., Dalian, China) from goat fetal fibroblasts and reverse-transcribed with the AMV 1st Strand cDNA Synthesis kit and an oligo $(\mathrm{dT})_{20}$ primer per the manufacturer's instructions (Takara Co. Ltd., China). An input of $1 \mu \mathrm{g}$ total RNA was used for each reaction.

The gVEGF164 cDNA was amplified by polymerase chain reaction (PCR) with cDNA as template at the appropriate annealing temperature for primers (forward: 5'ATGAACTTTCTGCTCTCT-3', reverse: 5'TCACCGCCTCGGCTTGTC-3') that contained BamH I (forward) and Hind III (reverse) restriction sites. The amplified cDNA fragment was cloned into pMD19-T (TaKaRa Co. Ltd., China), and the resulting plasmid, pMD19-gVEGF164, was transformed into E. coli DH5 $\alpha$ and sequenced on an ABI PRISM 377XL DNA Sequencer (Applied Biosystems, Inc. Foster City, CA, USA). Then, gVEGF164 was subcloned into the pET-his prokaryotic expression vector (Novagen, Inc. Madison, WI, USA) from pMD19-gVEGF164, generating the pET-gVEGF164 expression vector. The pET-gVEGF164 was transformed into $E$. coli BL21 (DE3) competent cells and confirmed by restriction analysis and sequencing.

\section{Expression of recombinant protein}

E. coli BL21 (DE3) cells were transformed with pETgVEGF164. The expression of $6 \times$ his-fused recombinant protein (6xhis-gVEGF164) was induced by $0.5 \mathrm{mM}$ isopropyl thio- $\beta$-D-galactoside (IPTG) for $5 \mathrm{~h}$ at $32^{\circ} \mathrm{C}$ to an $\mathrm{OD}_{600}$ of 0.6 . The expressed recombinant protein was identified by $15 \%(\mathrm{w} / \mathrm{v})$ sodium dodecyl sulfatepolyacrylamide gelelectrophoresis (SDS-PAGE). Premixed protein marker (TaKaRa Co. Ltd., China) was used as the molecular weight standard. Protein bands were visualized with Coomassie Brilliant Blue R-250 (Sigma-Aldrich, St. Louis, MO, USA), and protein content was measured by Bio-Rad assay (Bio-Rad Laboratories, Hercules, CA, USA).
The expressed recombinant protein was named rgVEGF164.

\section{Purification of recombinant goat VEGF164 and SDS- PAGE analysis}

The bacterial culture was harvested by centrifugation at $12,000 \mathrm{rpm}$ for $2 \mathrm{~min}$ at $4^{\circ} \mathrm{C}$, and the pellet was washed twice with $15 \mathrm{~mL}$ phosphate buffer saline (PBS) $(137 \mathrm{mM}$ $\mathrm{NaCl}, 2.7 \mathrm{mM} \mathrm{KCl}, 10 \mathrm{mM} \mathrm{Na}_{2} \mathrm{HPO}_{4}$, and $2 \mathrm{mM} \mathrm{KH}_{2} \mathrm{PO}_{4}$ ). The cells were dissolved in $2 \mathrm{~mL}$ cold $1 \times$ equilibration/wash buffer (50 mM sodium phosphate, 300 $\mathrm{mM} \mathrm{NaCl}$ ) with $0.75 \mathrm{mg} / \mathrm{mL}$ lysozyme, ultrasonicated, and centrifuged at $12,000 \mathrm{rpm}$ at $4^{\circ} \mathrm{C}$.

Recombinant rgVEGF164 was purified using the HisTALONGravity Columns Purification Kit (Clontech, Laboratories, Inc., Terra Bella Avenue, Mountain View, CA USA) per the manual, which is a His-tag nickel purification system. Then, the target protein was purified using the Micro Protein PAGE Recovery Kit (SangonBiotech Co., Ltd. Shanghai, China). Finally, the protein was dissolved in $0.1 \mathrm{M}$ phosphate buffer, $\mathrm{pH}$ 7.4. The protein lysate supernatants were electrophoresed on $15 \%(w / v)$ SDSpolyacrylamide gels, and unstained protein molecular weight marker (MBI Fermentas, Pittsburgh, PA, USA) was used as the molecular weight standard.

Western blot analysis of recombinant goat VEGF164 with monoclonal anti-6xhis and anti-vascular endothelial growth factor antibodies

Equal amounts $(30 \mu \mathrm{g})$ of purified protein were electrophoresed on $15 \%(\mathrm{w} / \mathrm{v})$ SDS-polyacrylamide gels. Then, the proteins were transferred to polyvinylidene fluoride (PVDF) membranes and incubated with monoclonal anti-His antibody (Cell Signaling Technology, Inc., Danvers, MA, USA) and monoclonal anti-VEGF antibody (Thermo Fisher Sientific Anatomical Pathology, Fremont, CA, USA) overnight at $4{ }^{\circ} \mathrm{C}$ and horseradish peroxidase -conjugated sheep anti-mouse IgG (GE Healthcare UK Limited, Dorset, UK) at room temperature for $1 \mathrm{~h}$. Signals were detected by chemiluminescence (Amersham).

Induction of hair growth by recombinant goat VEGF164

Eight-week-old wild-type Kunmingbai mice were used to monitor the induction of hair growth by rgVEGF164. 50 $\mu \mathrm{L}$ of $\mathrm{rgVEGF} 164$ ( $1 \mathrm{mg} / \mathrm{mL})$ was applied to a dorsal and lateral area of shaved skin, and control vehicle was applied with isopycnic phosphate-buffered saline to the opposing adjacent area on the same animal). Mice were painted with rgVEGF164 once a day for 1 week, and after that animals were fed for 2 weeks without treatment. The mice were housed under specific pathogen free (SPF) conditions and fed with SPF standards of care. 


\section{Statistical analysis}

Descriptive statistics were generated for all quantitative data, expressed as mean \pm SD. For each assay, triplicate parallel observations were examined.

\section{RESULTS}

Cloning of goat VEGF164 and construction of pETgVEGF164 expression vector

PCR of cDNA from goat fetal fibroblasts was performed to amplify a 573-bp fragment that encompassed the full open reading frame of gVEGF164, encoding 190 amino acids. The $26 \mathrm{~N}$-terminal amino acids constitute a signal peptide sequence. The open reading frame (ORF) fragment was amplified and cloned into pMD19-T. The resulting plasmid was designated pMD19-gVEGF164.

The pMD19-gVEGF164 was digested with BamH I and Hind III and subcloned into pET-his to obtain the pETgVEGF164 expression vector (Figure 1). The pETgVEGF164 was identified by restriction enzyme analysis, confirmed by sequencing, and transferred into E. coli BL21 (DE3) cells to express the $6 \times$ his-gVEGF164 recombinant protein.

Expression, purification, and identification of recombinant $6 \times$ his-gVEGF164 fusion protein

Goat VEGF164 was expressed as $6 \times$ his-gVEGF164 recombinant protein (rgVEGF164) after induction in $E$. coli BL21 (DE3) cells that were transformed with pETgVEGF164. We determined the optimal culture conditions for the rgVEGF164 protein as follows: $0.5 \mathrm{mM}$ IPTG with a 5 -h induction at 0.6 of the $O D_{600}$ value at $32^{\circ} \mathrm{C}$. The supernatants of the protein lysates from E. coli BL21 (DE3) cells, the bacterial cells transformed with pET-his and transformed with pET-gVEGF164 were electrophoresed on

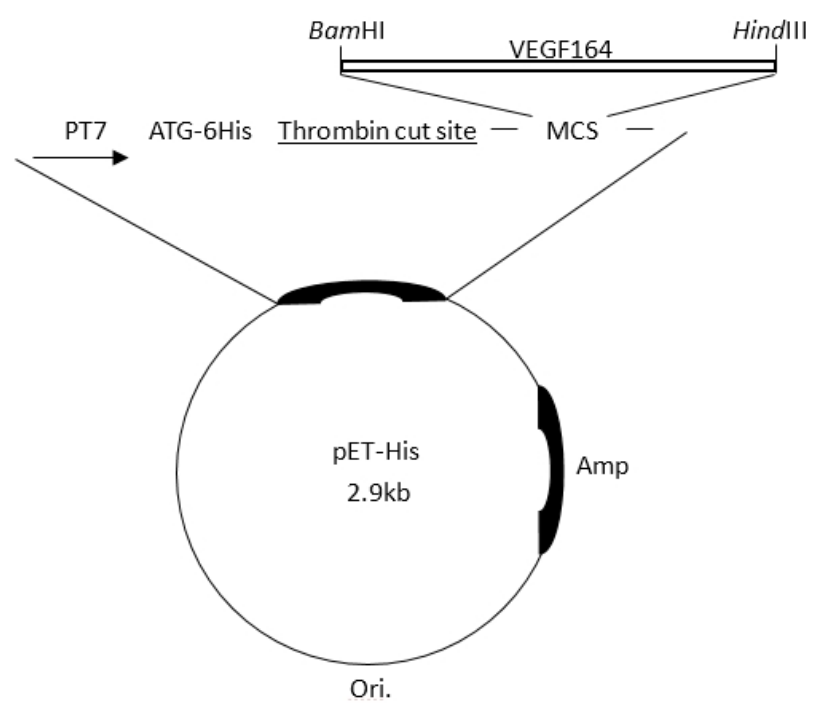

Figure 1. Schematic of insertion of goat VEGF164 cDNA into pET-his. VEGF, vascular endothelial growth factor.
$15 \%(w / v)$ SDS-polyacrylamide gels, and rgVEGF164 was detected by SDS-PAGE (Figure 2A)

The rgVEGF164 was purified from the total protein lysate of pET-gVEGF164-transformed bacteria and electrophoresed. A single band of approximately $26.0 \mathrm{kDa}$ appeared by $15 \%$ (w/v) SDS-PAGE (Figure 2B). After renaturation, the purified protein was identified by western blot using monoclonal anti-His (Figure 3A) and anti-VEGF antibodies (Figure 3B). Purified recombinant 6xhisgVEGF164 was then applied to the dorsal and lateral area of a shaved mouse.

\section{Recombinant goat VEGF164 increases hair growth in} mouse

To determine the bioactivity of rgVEGF164 in promoting hair growth, mice were treated with rgVEGF164. Mice were shaved, and rgVEGF164 was applied topically on the shaved area, as described in Methods. The treatment was administered for 7 days in the first week, and after that animals were fed for 2 weeks without treatment. As a result, rgVEGF164-treated, but not control, animals experienced rapid hair regrowth in 3 weeks (Figure 4), indicating that rgVEGF164 promotes hair growth.

\section{DISCUSSION}

The hair follicle, a type of epidermal appendage, is A

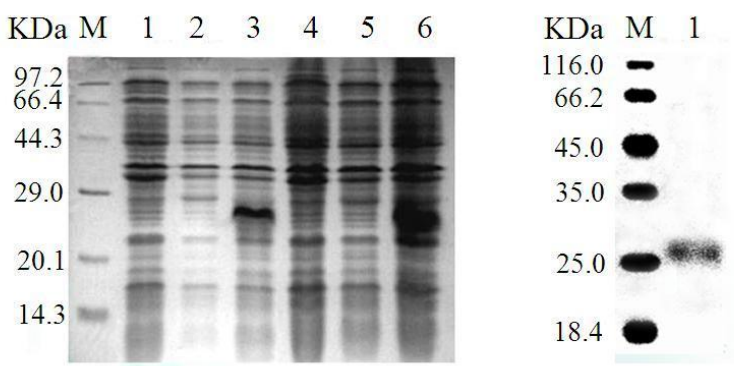

Figure 2. Analysis of the expression of rgVEGF164 in E. coli BL21 (DE3). (A) Expression of rgVEGF164 analyzed by $15 \%$ SDS-PAGE. Lane 1, lysates of E. coli BL21 (DE3) cells before induction; lane 2, lysates of BL21 cells harboring pET-His before induction; lane 3, lysates of BL21 cells harboring pET-gVEGF164 before induction; lane 4, lysates of BL21 cells after induction; lane 5, BL21 lysates harboring pET-His after induction; lane 6, lysates of BL21 cells harboring pET-gVEGF164 after induction; $M$, low protein molecular weight marker (D530S, Takara Co. Ltd., Dalian, China). The arrowhead indicates the target protein. (B) Purification and identification of rgVEGF164. Lane 1, purified VEGF164; $M$, unstained protein molecular weight marker (SM0431, Thermo Fisher Sientific Anatomical Pathology, Fremont, CA, USA). VEGF, vascular endothelial growth factor; rgVEGF164, recombinant goat VEFG164; SDS-PAGE, Sodium dodecyl sulfate-polyacrylamide gelelectrophoresis, gVEGF164, goat VEGF164. 
A

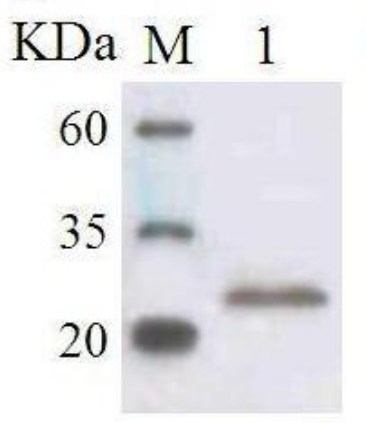

B

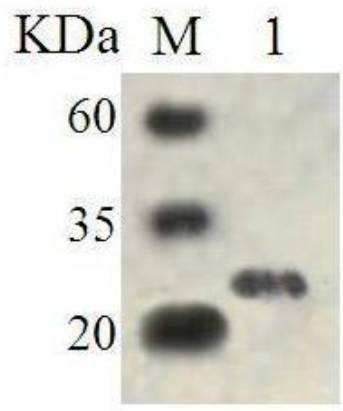

Figure 3. Western blot analysis of rgVEGF164. (A) Western blot analysis of rgVEGF164 with monoclonal anti-His antibody. Lane 1, purified rgVEGF164; $M$, PM Western Midview Marker (CW0021, Beijing ComWin Biotech Co. Ltd., Beijing, China). (B) Western blot analysis of rgVEGF164 with monoclonal anti-VEGF antibody. Lane 1, purified rgVEGF164; $M$, PM Western Midview Marker (CW0021, Beijing ComWin Biotech Co. Ltd., China) VEGF, vascular endothelial growth factor; rgVEGF164, recombinant goat VEFG164.

composed of dermal papillae cells, epithelial cells of the root sheath, and the hair shaft (Sperling, 1991). The hair follicle life cycle comprises the telogen, anagen, and catagen stages. Hair growth needs perifollicular vascularization, during which VEGF is upregulated in the outer root sheath (Yano et al., 2001) and highly expressed in anagen hair follicles, including the outer and inner root sheaths and dermal papillae epidermal matrix, particularly in the follicular basement membrane zone in normal human skin (Man et al., 2009). The VEGF promotes hair growth, increases the number of hair follicles, and elongates the hair shaft by improving follicle vascularization in mice (Yano et al., 2001; Ozeki and Tabata, 2002).

The effects of VEGF are mediated primarily through its receptors, VEGF receptor-1 (fms-like tyrosine kinase-1), -2

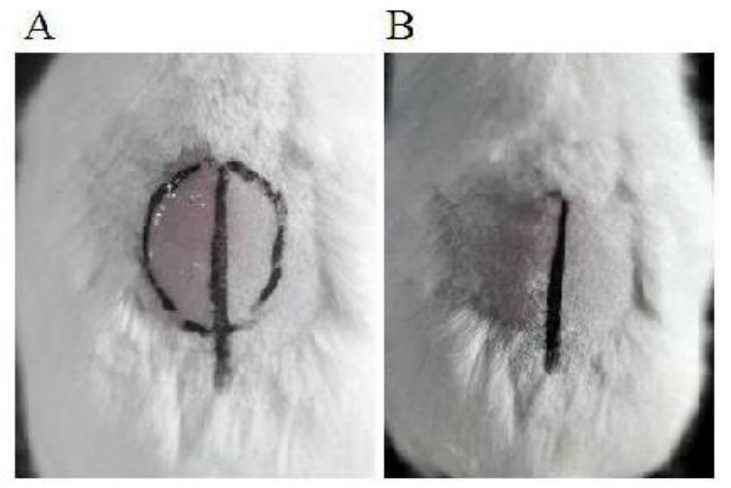

Figure 4. Gross appearance of skin from control and rgVEGF164treated sides of mice. After 3 weeks, rgVEGF164-treated animals displayed quick hair regrowth. Right side is rgVEGF164-treated side and left side is control. VEGF, vascular endothelial growth factor; rgVEGF164, recombinant goat VEFG164. (kinase domain region), and -3 (soluble Flt). The chief receptor of VEGF, VEGFR-2, is believed to mediate most processes, such as cell proliferation, migration, and survival (Ferrara et al., 2003; Holmes et al., 2007; Olsson et al., 2006). Basal cells of the sebaceous glands expressed abundant VEGF and VEGFR-2 (Man et al., 2009), and VEGF active VEGFR-2 in the hair follicle stem cell (Wu et al., 2014), and VEGF165 upregulates VEGFR-2 mRNA and protein and induces phosphorylation of VEGFR-2 in the outer root sheath. Exogenous VEGF stimulates VEGFR-2mediated proliferation through phosphorylation of ERK in human outer root sheath cells and human hair follicle dermal papilla cells ( $\mathrm{Li}$ et al., 2012a).

The VEGF-overexpressing transgenic mice experience accelerated hair regrowth compared with wild-type littermates, and have longer and thicker hair after 11 days (Yano, 2001). In contrast, abdominal fur does not regrow properly in rats that have been treated with AEE788, a dual inhibitor of epidermal growth factor receptor (EGFR) and VEGFR, over 4 weeks (Deng et al., 2011). These results demonstrate that VEGF mediates hair growth during development.

In this study, we generated functional rgVEGF164 protein using a prokaryotic expression system, wherein an approximately $26-\mathrm{kDa}$ recombinant protein was detected by monoclonal anti-VEGF. The VEGF has a molecular weight of roughly $45 \mathrm{kDa}$ under nonreducing conditions versus about $23 \mathrm{kDa}$ under reducing conditions (Ferrara and Henzel, 1989). The VEGF is a homodimer that comprises 2 polypeptide chains and has a molecular mass of approximately $45 \mathrm{kDa}$; the single stranded form has a molecular weight of about $23 \mathrm{kDa}$ under denaturing conditions. We believe that the 6xhis-gVEGF164 fusion protein exists in monomeric form-rgVEGF164 can not form a homodimer due to the use of a prokaryotic expression system. Renatured rgVEGF164 was smeared across a dorsal area of a shaved mouse and had a positive effect on hair growth. These results demonstrate that rgVEGF164 increases hair growth in mice.

\section{CONCLUSION}

We cloned goat VEGF and expressed it in E. coli BL21 (DE3) cells as a $6 \times$ his-tagged fusion protein. rgVEGF was purified and identified by western blot using monoclonal anti-VEGF. The rgVEGF164 was applied to the dorsal area of a shaved mouse, accelerating hair regrowth faster than in the control group. Thus, rgVEGF164 effects hair growth in mice.

\section{ACKNOWLEDGMENTS}

This work was supported by a grants from the Natural 
Sciences Foundation of China (No. 31160469, 31360561); Natural Sciences Foundation of Inner Mongolia, China (No. 2011MS0521); a graduate student research project of Inner Mongolia University; and Major Projects for New Varieties of Genetically Modified Organisms (No.2013ZX08008002).

\section{REFERENCES}

Carmeliet, P., V. Ferreira, G. Breier, S. Pollefeyt, L. Kieckens, M. Gertsenstein, M. Fahrig, A. Vandenhoeck, K. Harpal, C. Eberhardt, C. Declercq, J. Pawling, L. Moons, D. Collen, W. Risau, and A. Nagy. 1996. Abnormal blood vessel development and lethality in embryos lacking a single VEGF allele. Nature 380:435-439.

Deng, M., H. Huang, H. Jin, O. Dirsch, and U. Dahmen. 2011. The anti-proliferative side effects of AEE788, a tyrosine kinase inhibitor blocking both EGF- and VEGF-receptor, are liversize-dependent after partial hepatectomy in rats. Invest. New Drugs 29:593-606.

Ferrara, N., H. P. Gerber, and J. LeCouter. 2003. The biology of VEGF and its receptors. Nat. Med. 9:669-676.

Ferrara N. and W. J. Henzel. 1989. Pituitary follicular cells secrete a novel heparin-binding growth factor specific for vascular endothelial cells. Biochem. Biophys. Res. Commun. 161:851858

Ford, K. M. and P. A. D'Amore. 2012. Molecular regulation of vascular endothelial growth factor expression in the retinal pigment epithelium. Mol. Vis. 18:519-527.

Gordon, O., D. Gilon, Z. He, D. May, A. Lazarus, A. Oppenheim, and E. Keshet. 2012. Vascular endothelial growth factorinduced neovascularization rescues cardiac function but not adverse remodeling at advanced ischemic heart disease. Arterioscler. Thromb. Vasc. Biol. 32:1642-1651.

Gospodarowicz, D., J. A. Abraham, and J. Schilling. 1989. Isolation and characterization of a vascular endothelial cell mitogen produced by pituitary-derived folliculo stellate cells. Proc. Natl. Acad. Sci. US Aa. 86:7311-7315.

Holmes, K., O. L. Roberts, A. M. Thomas, and M. J. Cross. 2007. Vascular endothelial growth factor receptor-2: structure, function, intracellular signalling and therapeutic inhibition. Cell. Signal. 19:2003-2012.

Jingjing, L., Y. Xue, N. Agarwal, and R. S. Roque. 1999. Human Muller cells express VEGF183, a novel spliced variant of vascular endothelial growth factor. Invest. Ophthalmol. Vis. Sci. 40:752-759.

Li, W., Z. F. Lu, X. Y. Man, C. M. Li, J. Zhou, J. Q. Chen, X. H. Yang, X. J. Wu, S. Q. Cai, and M. Zheng. 2012a. VEGF upregulates VEGF receptor-2 on human outer root sheath cells and stimulates proliferation through ERK pathway. Mol. Biol. Rep. 39:8687-8694.

Li, W., X. Y. Man, C. M. Li, J. Q. Chen, J. Zhou, S. Q. Cai, Z. F. $\mathrm{Lu}$, and M. Zheng. 2012b. VEGF induces proliferation of human hair follicle dermal papilla cells through VEGFR-2mediated activation of ERK. Exp. Cell Res. 318:1633-1640.

Li, X. D., J. Chen, C. C. Ruan, D. L. Zhu, and P. J. Gao. 2012c. Vascular endothelial growth factor-induced osteopontin expression mediates vascular inflammation and neointima formation via Flt-1 in adventitial fibroblasts. Arterioscler. Thromb. Vasc. Biol. 32:2250-2258.

Liu, Y., A. D. Berendsen, S. Jia, S. Lotinun, R. Baron, N. Ferrara, and B. R. Olsen. 2012. Intracellular VEGF regulates the balance between osteoblast and adipocyte differentiation. J. Clin. Invest. 122:3101-3113.

Magnuson, J., F. Leonessa, and G. S. Ling. 2012. Neuropathology of explosive blast traumatic brain injury. Curr. Neurol. Neurosci. Rep. 12:570-579.

Man, X. Y., X. H. Yang, S. Q. Cai, Z. Y. Bu, X. J. Wu, Z. F. Lu, and M. Zheng. 2009. Expression and localization of vascular endothelial growth factor and vascular endothelial growth factor receptor-2 in human epidermal appendages: a comparison study by immunofluorescence. Clin. Exp. Dermatol. 34:396-401.

Olsson, A. K., A. Dimberg, J. Kreuger, and L. Claesson-Welsh. 2006. VEGF receptor signalling - in control of vascular function. Nat. Rev. Mol. Cell Biol. 7:359-371.

Ozeki, M. and Y. Tabata. 2002. Promoted growth of murine hair follicles through controlled release of vascular endothelial growth factor. Biomaterials 23:2367-2373.

Poltorak, Z., T. Cohen, R. Sivan, Y. Kandelis, G. Spira, I. Vlodavsky, E. Keshet, and G. Neufeld. 1997. VEGF145, a secreted vascular endothelial growth factor isoform that binds to extracellular matrix. J. Biol. Chem. 272:7151-7158.

Senger, D. R., L. Van de Water, L. F. Brown, J. A. Nagy, K. T. Yeo, T. K. Yeo, B. Berse, R. W. Jackman, A. M. Dvorak, and H. F. Dvorak. 1993. Vascular permeability factor (VPF, VEGF) in tumor biology. Cancer Metastasis Rev. 12:303-324.

Sitohy, B., J. A. Nagy, and H. F. Dvorak. 2012. AntiVEGF/VEGFR therapy for cancer: Reassessing the target. Cancer Res. 72:1909-1914.

Sperling, L. C. 1991. Hair anatomy for the clinician. J. Am. Acad. Dermatol. 25:1-17.

Tischer, E., R. Mitchell, T. Hartman, M. Silva, D. Gospodarowicz, J. C. Fiddes, and J. A. Abraham. 1991. The human gene for vascular endothelial growth factor. Multiple protein forms are encoded through alternative exon splicing. J. Biol. Chem. 266:11947-11954.

Whittle, C., K. Gillespie, R. Harrison, P. W. Mathieson, and S. J. Harper. 1999. Heterogeneous vascular endothelial growth factor (VEGF) isoform mRNA and receptor mRNA expression in human glomeruli, and the identification of VEGF148 mRNA, a novel truncated splice variant. Clin. Sci. (Lond). 97:303-312.

Wu, X. J., J. W. Zhu, J. Jing, D. Xue, H. Liu, M. Zheng, and Z. F. Lu. 2014. VEGF165 modulates proliferation, adhesion, migration and differentiation of cultured human outer root sheath cells from central hair follicle epithelium through VEGFR-2 activation in vitro. J. Dermatol. Sci. 73:152-160.

Yano, K., L. F. Brown, and M. Detmar. 2001. Control of hair growth and follicle size by VEGF-mediated angiogenesis. J. Clin. Invest. 107:409-417

Zhang, Q. L., J. P. Li, Y. M. Li, Q. Chang, Y. Chen, H. Z. Jiang, Z. H. Zhao, and D. Guo. 2013. Expression and localization of the vascular endothelial growth factor and changes of microvessel density during hair follicle development of liaoning cashmere goats. Genet. Mol. Res. 12:6424-6432. 